\title{
Electronic structure of spheroidal fullerenes in a weak uniform magnetic field: a continuum field-theory model
}

\author{
M. Pudlak ${ }^{a}$, R. Pincak ${ }^{a, b}$ and V.A. Osipov ${ }^{b}$ \\ ${ }^{a}$ Institute of Experimental Physics, Slovak Academy of Sciences, Watsonova 47,04353 Kosice, Slovak Republic \\ ${ }^{b}$ Joint Institute for Nuclear Research, Bogoliubov Laboratory of Theoretical Physics, 141980 Dubna, Moscow region, Russia \\ e-mail:pudlak@saske.sk,pincak@saske.sk, osipov@thsun1.jinr.ru
}

(July 17, 2021)

\begin{abstract}
The effect of a weak uniform magnetic field on the electronic structure of slightly deformed fullerene molecules is studied within the continuum field-theory model. It is shown how the existing due to spheroidal deformation fine structure of the electronic energy spectrum modifies in the presence of the magnetic field. Exact analytical solutions for zero-energy modes are found.
\end{abstract}

\section{INTRODUCTION}

Recently, we have considered the problem of the low energy electronic states in spheroidal fullerenes [1]. The main findings were a discovery of fine structure with a specific shift of the electronic levels upwards due to spheroidal deformation. In addition, three twofold degenerate modes near the Fermi level with one of them being the true zero mode were found. An interesting question is the modification of this structure under the influence of a uniform magnetic field.

The problem of Zeeman splitting and Landau quantization of electrons on a sphere was studied in Ref. [2]. To this end, the Schrödinger equation for a free electron on the surface of a sphere in a uniform magnetic field was formulated and solved. In this paper, we explore the field-theory model suggested in Ref. [1], which describes the electronic states near the Fermi energy and takes into account the specific structure of carbon lattice, geometry, and the topological defects (pentagons). The Euler's theorem for graphene requires the presence of twelve pentagons to get the closed molecule. In the framework of continuum description we extend the Dirac operator by introducing the Dirac monopole field inside the spheroid to simulate the elastic vortices due to twelve pentagonal defects. The K spin flux which describes the exchange of two different Dirac spinors in the presence of a conical singularity is included in a form of t'Hooft-Polyakov monopole. Our studies cover slightly elliptically deformed molecules in the weak uniform external magnetic field.

\section{THE MODEL}

Let us start with writing down the Dirac operator for free massless fermions on the Riemannian spheroid $S^{2}$. The Dirac equation on a surface $\Sigma$ in the presence of the abelian magnetic monopole field $W_{\mu}$ and the external magnetic field $A_{\mu}$ is written as [3]

$$
i \gamma^{\alpha} e_{\alpha}^{\mu}\left[\nabla_{\mu}-i W_{\mu}-i A_{\mu}\right] \psi=E \psi,
$$

where $e_{\alpha}^{\mu}$ is the zweibein, $g_{\mu \nu}=e_{\mu}^{\alpha} e_{\nu}^{\beta} \delta_{\alpha \beta}$ is the metric, the orthonormal frame indices $\alpha, \beta=\{1,2\}$, the coordinate indices $\mu, \nu=\{1,2\}$, and $\nabla_{\mu}=\partial_{\mu}+\Omega_{\mu}$ with

$$
\Omega_{\mu}=\frac{1}{8} \omega_{\mu}^{\alpha}{ }_{\mu}^{\beta}\left[\gamma_{\alpha}, \gamma_{\beta}\right]
$$

being the spin connection term in the spinor representation (see [1] for details). The energy in (1) is measured from the Fermi level.

\section{A. Zero-energy mode}

Let us start from the analysis of an electron state at the Fermi level (so-called zero-energy mode). We will use the projection coordinates in the form 


$$
x=\frac{2 R^{2} r}{R^{2}+r^{2}} \cos \phi ; \quad y=\frac{2 R^{2} r}{R^{2}+r^{2}} \sin \phi ; \quad z=-c \frac{R^{2}-r^{2}}{R^{2}+r^{2}},
$$

where $R$ and $c$ are the spheroidal axles. The Riemannian connection with respect to the orthonormal frame is written as $[4,5]$

$$
-\omega_{\phi 2}^{1}=\omega_{\phi 1}^{2}=\frac{R^{2}-r^{2}}{\sqrt{\left(R^{2}-r^{2}\right)^{2}+4 c^{2} r^{2}}} ; \quad \omega_{r 2}^{1}=\omega_{r 1}^{2}=0 .
$$

The only nonzero component of the gauge field $W_{\mu}$ in region $R_{N}$ for spheroidal fullerenes reads (see Ref. [1])

$$
W_{\phi}=G+m \frac{z}{X}
$$

where

$$
X=\frac{\sqrt{c^{2}\left(r^{2}-R^{2}\right)^{2}+4 R^{4} r^{2}}}{r^{2}+R^{2}} .
$$

Notice that the monopole field $W_{\mu}$ in Eq. (5) consists of two parts. The first one comes from the K-spin connection term and implies the charge $g= \pm 3 / 2$ while the second one is due to elastic flow through a surface. This contribution is topological in its origin and characterized by charge $G$. For total elastic flux from twelve pentagonal defects one has $G=1$. The parameter $m=g-G$ is introduced in Eq.(5). The uniform external magnetic field $B$ is chosen to be pointed in the $z$ direction so that $\vec{A}=B(y,-x, 0) / 2$. In projection coordinates the only nonzero component of $A_{\mu}$ is written as

$$
A_{\phi}=-\frac{2 B R^{4} r^{2}}{\left(R^{2}+r^{2}\right)^{2}}
$$

Let us study Eq.(1) for the electronic states at the Fermi energy $(E=0)$. The Dirac matrices can be chosen to be the Pauli matrices, $\gamma_{1}=-\sigma_{2}, \gamma_{2}=-\sigma_{1}$. By using the substitution

$$
\left(\begin{array}{c}
\psi_{A} \\
\psi_{B}
\end{array}\right)=\sum_{j} \frac{e^{i(j+G) \phi}}{\sqrt{2 \pi}}\left(\begin{array}{c}
u_{j}(r) \\
v_{j}(r)
\end{array}\right), j=0, \pm 1, \pm 2, \ldots
$$

we obtain

$$
\begin{aligned}
& \left(\frac{1}{r}\left(j-m \frac{z}{X}-A_{\phi}\right)+\frac{R^{2}+r^{2}}{K}\left(\partial_{r}-\frac{r^{2}-R^{2}}{2 r\left(r^{2}+R^{2}\right)}\right)\right) v(r)=0 \\
& \left(\frac{1}{r}\left(j-m \frac{z}{X}-A_{\phi}\right)-\frac{R^{2}+r^{2}}{K}\left(\partial_{r}-\frac{r^{2}-R^{2}}{2 r\left(r^{2}+R^{2}\right)}\right)\right) u(r)=0,
\end{aligned}
$$

where $K=\sqrt{\left(R^{2}-r^{2}\right)^{2}+4 c^{2} r^{2}}$. We assume that the eccentricity of the spheroid is small enough. In this case, one can write down $c=R+\delta R$ and $\delta(|\delta| \ll 1)$ is a small dimensionless parameter characterizing the spheroidal deformation. Therefore, one can follow the perturbation scheme using $\delta$ as the perturbation parameter. To the leading in $\delta$ approximation Eqs. (11) and (12) are written as

$$
\begin{aligned}
\partial_{r} v_{j}(r)=(- & \frac{\left(R^{2}+r^{2}\right)^{2}}{r\left[\left(R^{2}+r^{2}\right)^{2}-4 R^{2} r^{2} \delta\right]}\left[j-m(1+\delta) \frac{r^{2}-R^{2}}{r^{2}+R^{2}}+m \delta \frac{\left(r^{2}-R^{2}\right)^{3}}{\left(r^{2}+R^{2}\right)^{3}}\right] \\
& \left.-\frac{2 B R^{4} r}{\left[\left(R^{2}+r^{2}\right)^{2}-4 R^{2} r^{2} \delta\right]}+\frac{r^{2}-R^{2}}{2 r\left(R^{2}+r^{2}\right)}\right) v_{j}(r), \\
\partial_{r} u_{j}(r)=( & \frac{\left(R^{2}+r^{2}\right)^{2}}{r\left[\left(R^{2}+r^{2}\right)^{2}-4 R^{2} r^{2} \delta\right]}\left[j-m(1+\delta) \frac{r^{2}-R^{2}}{r^{2}+R^{2}}+m \delta \frac{\left(r^{2}-R^{2}\right)^{3}}{\left(r^{2}+R^{2}\right)^{3}}\right] \\
& \left.+\frac{2 B R^{4} r}{\left[\left(R^{2}+r^{2}\right)^{2}-4 R^{2} r^{2} \delta\right]}+\frac{r^{2}-R^{2}}{2 r\left(R^{2}+r^{2}\right)}\right) u_{j}(r) .
\end{aligned}
$$


The exact solution to Eqs. (11) and (12) is found to be

$$
\begin{gathered}
v_{j}(x)=\frac{(x+1)^{(1 / 2-m)}}{x^{\frac{1}{2}(j+m+1 / 2)}}\left(\frac{x+1-2 \delta-2 \sqrt{\delta(\delta-1)}}{x+1-2 \delta+2 \sqrt{\delta(\delta-1)}}\right)^{-\alpha}\left((x+1)^{2}-4 \delta x\right)^{m}, \\
u_{j}(x)=(x+1)^{(m+1 / 2)} x^{\frac{1}{2}(j+m-1 / 2)}\left(\frac{x+1-2 \delta-2 \sqrt{\delta(\delta-1)}}{x+1-2 \delta+2 \sqrt{\delta(\delta-1)}}\right)^{\alpha}\left((x+1)^{2}-4 \delta x\right)^{-m},
\end{gathered}
$$

where $x=r^{2} / R^{2}, \alpha=(j / 2) \sqrt{\delta /(\delta-1)}+B R^{2} / 4 \sqrt{\delta(\delta-1)}$. The function $u_{j}$ can be normalized if the condition $m-1 / 2<j<1 / 2-m$ is fulfilled. In turn, the normalization condition for $v_{j}$ has the form $-1 / 2-m<j<m+1 / 2$. Finally, there are five normalized solutions for $u_{j}$ with $j=0, \pm 1, \pm 2$ when $m=-5 / 2$, and one solution for $v_{j}$ with $j=0$ when $m=1 / 2$. In the limit $\delta \rightarrow 0$ we arrive at the solution for spherical fullerenes

$$
\begin{gathered}
v_{j}(r)=r^{(-1 / 2-j-m)}\left(R^{2}+r^{2}\right)^{(1 / 2+m)} e^{\frac{B R^{4}}{R^{2}+r^{2}}} \\
u_{j}(r)=r^{(-1 / 2+j+m)}\left(R^{2}+r^{2}\right)^{(1 / 2-m)} e^{-\frac{B R^{4}}{R^{2}+r^{2}}} .
\end{gathered}
$$

Since normalization conditions do not depend on the parameter $\delta$ they are the same as for spherical case. Thus the perturbation does not change the number of zero modes. Notice that Eq. (15) can be obtained from Eqs. (13) and (14) by using $\lim [1+(x / a)]^{a}=e^{x}, a \rightarrow \infty$.

\section{B. Electronic states near the Fermi energy}

Let us study now the structure of electron levels near the Fermi energy. It is convenient to consider this problem by using of the Cartesian coordinates $x, y, z$ in the form

$$
x=a \sin \theta \cos \phi ; \quad y=a \sin \theta \sin \phi ; \quad z=c \cos \theta
$$

The Riemannian connection reads (cf. (4))

$$
\omega_{\phi 2}^{1}=-\omega_{\phi 1}^{2}=\frac{a \cos \theta}{\sqrt{a^{2} \cos ^{2} \theta+c^{2} \sin ^{2} \theta}} ; \quad \omega_{\theta 2}^{1}=\omega_{\theta 1}^{2}=0 .
$$

Within the framework of the perturbation scheme the spin connection coefficients are written as

$$
\omega_{\phi 2}^{1}=-\omega_{\phi 1}^{2} \approx \cos \theta\left(1-\delta \sin ^{2} \theta\right)
$$

where the terms to first order in $\delta$ are taken into account. In spheroidal coordinates, the only nonzero component of $W_{\mu}$ in region $R_{N}$ is found to be (see Ref. [1])

$$
W_{\phi} \approx g \cos \theta\left(1+\delta \sin ^{2} \theta\right)+G(1-\cos \theta)-\delta G \sin ^{2} \theta \cos \theta,
$$

and the external magnetic field reads

$$
A_{\phi}=-\frac{1}{2} B a^{2} \sin ^{2} \theta
$$

By using the substitution (8) we obtain the Dirac equation for functions $u_{j}$ and $v_{j}$ in the form

$$
\left(-i \sigma_{1} \frac{1}{a}\left(\partial_{\theta}+\frac{\cot \theta}{2}\right)+\frac{\sigma_{2}}{a \sin \theta}\left(j-m \cos \theta+\frac{1}{2} B a^{2} \sin ^{2} \theta\right)+\delta \hat{\mathcal{D}}_{1}\right)\left(\begin{array}{c}
u_{j}(\theta) \\
v_{j}(\theta)
\end{array}\right)=E\left(\begin{array}{c}
u_{j}(\theta) \\
v_{j}(\theta)
\end{array}\right)
$$

where

$$
\hat{\mathcal{D}}_{1}=-\frac{\gamma_{1}}{a} \sin \theta(j-2 m \cos \theta)-\gamma_{1} \frac{B a}{2} \sin ^{3} \theta
$$


A convenient way to study the eigenvalue problem is to square Eq. (21). For this purpose, let us write the Dirac operator in Eq. (21) as $\hat{\mathcal{D}}=\hat{\mathcal{D}}_{0}+\delta \hat{\mathcal{D}}_{1}$. One can easily obtain that (similar way as in Ref. [6])

$$
\begin{array}{r}
\hat{\mathcal{D}}_{0}^{2}=-\frac{1}{a^{2}}\left(\partial_{\theta}^{2}+\frac{\cos \theta}{\sin \theta} \partial_{\theta}-\frac{1}{4}-\frac{1}{4 \sin ^{2} \theta}\right)+\frac{(j-m \cos \theta)^{2}}{a^{2} \sin ^{2} \theta}+B j+\frac{B}{2}\left(\sigma_{3}-2 m\right) \cos \theta \\
+\sigma_{3} \frac{m-j \cos \theta}{a^{2} \sin ^{2} \theta}+\frac{B^{2} a^{2} \sin ^{2} \theta}{4} .
\end{array}
$$

Notice that to first order in $\delta$ the square of the Dirac operator is written as $\hat{\mathcal{D}}^{2}=\left(\hat{\mathcal{D}}_{0}^{2}+\delta \hat{\Gamma}\right)$, where $\hat{\Gamma}=\left(\hat{\mathcal{D}}_{0} \hat{\mathcal{D}}_{1}+\hat{\mathcal{D}}_{1} \hat{\mathcal{D}}_{0}\right)$. In an explicit form

$$
\begin{gathered}
a^{2} \hat{\Gamma}=2 j^{2}+j x\left(\sigma_{3}-6 m\right)+4 m\left(m-\sigma_{3}\right) x^{2}+2 m \sigma_{3}+3 B a^{2}\left(1-x^{2}\right) x\left(\sigma_{3} / 2-m\right) \\
+2 B j a^{2}\left(1-x^{2}\right)+B^{2} a^{4}\left(1-x^{2}\right)^{2} / 2
\end{gathered}
$$

where the appropriate substitution $x=\cos \theta$ is used. The equation $\hat{\mathcal{D}}^{2} \psi=E^{2} \psi$ takes the form

$$
\begin{gathered}
{\left[\partial_{x}\left(1-x^{2}\right) \partial_{x}-\frac{(j-m x)^{2}-j \sigma_{3} x+\frac{1}{4}+\sigma_{3} m}{1-x^{2}}-a^{2} B V(x)-\delta a^{2} \hat{\Gamma}\right]\left(\begin{array}{c}
u_{j}(x) \\
v_{j}(x)
\end{array}\right)} \\
=-\left(\lambda^{2}-\frac{1}{4}\right)\left(\begin{array}{c}
u_{j}(x) \\
v_{j}(x)
\end{array}\right)
\end{gathered}
$$

where $\lambda=a E, V(x)=j+\left(\sigma_{3}-2 m\right) x / 2$. Since we consider the case of a weak magnetic field the terms with $B^{2}$ and $\delta B$ in Eq. (25) can be neglected. As a result, the energy spectrum for spheroidal fullerenes is found to be

$$
\left(\lambda_{j n}^{\delta}\right)^{2}=(n+|j|+1 / 2)^{2}-m^{2}+B a^{2} j+B a^{2} A_{j n}+\delta F(j, n, m)
$$

where

$$
A_{j n}=-\frac{j\left(m^{2}-1 / 4\right)}{p(p+1)}
$$

The function of spheroidal deformations $F(j, n, m)$ is specified in Ref. [1]. Finally, in the linear in $\delta$ approximation, the low energy electronic spectrum of spheroidal fullerenes takes the form

$$
E_{j n}^{\delta}=E_{j n}^{0}+E_{j n}^{0 B_{z}}+E_{j n}^{\delta}
$$

with

$$
E_{j n}^{0}= \pm \sqrt{(2 \xi+n)(2 \eta+n)}, \quad E_{j n}^{0 B_{z}}=\frac{B a^{2}\left(j+A_{j n}\right)}{2 E_{j n}^{0}}, E_{j n}^{\delta}=\frac{\delta F(j, n, m)}{2 E_{j n}^{0}},
$$

where $\xi=\mu(\nu)$ and $\eta=\beta(\alpha)$ for $j>0(j<0)$, respectively. Here we came back to the energy variable $E=\lambda / a$ (in units of $\hbar V_{F} / a$ where $V_{F}$ is the Fermi velocity).

As is seen from Eq. (27), for $B=0$ the spheroidal deformation gives rise to an appearance of a additional structure. As an example, Table 1 shows all five contributions (in compliance with Eq. (27)) to the first and second energy levels for $\mathrm{YO}_{-} \mathrm{C}_{240}$ ( $\mathrm{YO}$ means a structure given in $[7,8]$ ). As is seen, the energy levels become shifted due to spheroidal deformation. The uniform magnetic field provides the well-known Zeeman splitting. The difference between topological and Zeeman splitting is clearly seen. In the second case, the splitted levels are shifted in opposite directions while for topological splitting the shift is always positive. As an illustration, Fig. 1 schematically shows the structure of the second level. In this case, the initial (for $\delta=0)$ degeneracy of $E_{j n}^{0}$ is equal to six. The spheroidal deformation provokes an appearance of three shifted double degenerate levels (fine structure). The magnetic field is responsible to Zeeman splitting.

We admit that the obtained values of the splitting energies can deviate from the estimations within some more precise microscopic lattice models and density-functional methods (see, e.g. [9]). In our paper, we focused mostly on the very existence of physically interesting effects. For this reason, the values in both Table and the schematic picture are presented with accuracy at about one percent of $E_{j n}^{0}$. 


\section{CONCLUSION}

In conclusion, we have considered the structure of low energy electronic states of spheroidal fullerenes in the weak uniform magnetic field provided the spheroidal deformation from the sphere is small enough. For the states at the Fermi level, we found an exact solution for the wave functions. It is shown that the external magnetic field modifies the density of electronic states and does not change the number of zero modes. For non-zero energy modes, electronic states near the Fermi energy of spheroidal fullerene are found to be splitted in the presence of a weak uniform magnetic field. It should be mentioned that the zero-energy states in our model corresponds to the HOMO (highest occupied molecular orbital) states in the calculations based on the local-density approximation in the density-functional theory (see, e.g. [10]). In particular, the HOMO-LUMO energy gap is found to be about $1.1 \mathrm{eV}$ for YO-C 240 fullerene within our model (here LUMO means the lowest unoccupied molecular orbital).

The work was supported in part by VEGA grant 2/7056/27. of the Slovak Academy of Sciences, by the Science and Technology Assistance Agency under contract No. APVT-51-027904 and by the Russian Foundation for Basic Research under Grant No. 05-02-17721.

[1] M. Pudlak, R. Pincak and V.A. Osipov, Phys. Rev. B 74 (2006) 235435.

[2] H. Aoki and H. Suezawa, Phys. Rev. A 46 (1992) R1163.

[3] N.D. Birrell and P.C.W. Davies, Quantum Fields in Curved Space, (Cambridge 1982).

[4] M. Nakahara, Geometry, Topology and Physics, (Institute of Physics Publishing Bristol 1998).

[5] M.Göckeler and T.Schücker, Differential geometry, gauge theories, and gravity (Cambridge University Press 1989).

[6] D.V. Kolesnikov and V.A. Osipov, Eur.Phys.Journ. B 49 (2006) 465.

[7] M. Yoshida and E. Osawa, Fullerene Sci. Tech. 1 (1993) 55.

[8] J. P. Lu and W. Yang, Phys. Rev. B 49 (1994) 11421.

[9] R.A. Broglia, G. Colò, G. Onida, H.E. Roman, Solid State Physics of Finite Systems, (Springer 2004).

[10] S. Saito and A. Oshiyama, Phys. Rev. Lett. 66 (1991) 2637. 


\begin{tabular}{|c|c|c|c|c|}
\hline$Y O-C_{240} \quad B a^{2}=0.1$ & $j$ & $E_{j n}^{0}(e V)$ & $E_{j n}^{0 B_{z}}(m e V)$ & $E_{j n}^{\delta}(m e V)$ \\
\hline \multirow[t]{2}{*}{$n=0, m=1 / 2$} & 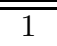 & 1.094 & 27 & 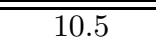 \\
\hline & -1 & 1.094 & -27 & 10.5 \\
\hline \multirow[t]{2}{*}{$\bar{n}=1, m=1 / 2$} & 1 & 1.89 & 16 & 8.8 \\
\hline & -1 & 1.89 & -16 & 8.8 \\
\hline \multirow[t]{2}{*}{$n=0, m=1 / 2$} & 2 & 1.89 & 32 & 28.4 \\
\hline & -2 & 1.89 & -32 & 28.4 \\
\hline \multirow[t]{2}{*}{$n=0, m=-5 / 2$} & 3 & 1.89 & 24 & 3 \\
\hline & -3 & 1.89 & -24 & 3 \\
\hline
\end{tabular}

TABLE I. The structure of the first and higher energy levels for $\mathrm{YO}-\mathrm{C}_{240}$ fullerene in uniform magnetic field. The hopping integral and other parameters are taken to be $t=2.5 \mathrm{eV}$ and $V_{F}=3 t \bar{b} / 2 \hbar, \bar{b}=1.45 \AA, \bar{R}=7.03 \AA, S D=0.17 \AA, \delta=0.024$. $\bar{b}$ is average bond length, $\bar{R}(\bar{R}=a)$ is average radius, $S D$ is standard deviation from a perfect sphere (see Refs. $[7,8])$, so that $\delta=S D / \bar{R}$.

$$
E_{j n}^{0} \quad+E_{j n}^{\delta} \quad+E_{j n}^{0 B_{z}}
$$

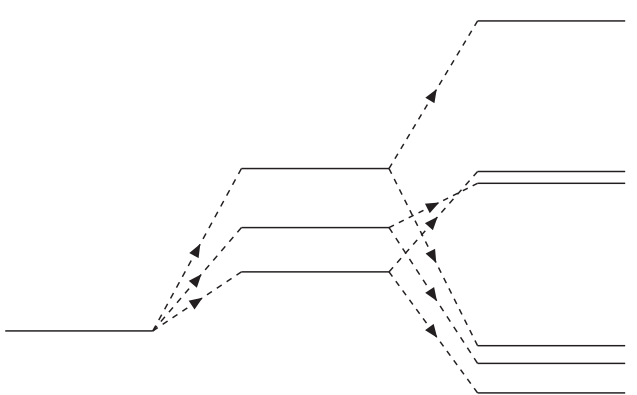

FIG. 1. The schematic picture of the second positive electronic level $\underbrace{s p h e r o i d a l ~ f u l l e r e n e s ~ i n ~ a ~ w e a k ~ u n i f o r m ~}_{E_{j n}^{\delta} \text { of the }}$ magnetic field. 